\title{
Analysis of the technical biases of meteor video cameras used in the CILBO system
}

\author{
Thomas Albin $^{1,2}$, Detlef Koschny ${ }^{3,4}$, Sirko Molau ${ }^{5}$, Ralf Srama $^{1}$, and Björn Poppe ${ }^{2}$ \\ ${ }^{1}$ Institute of Space Systems, University of Stuttgart, Pfaffenwaldring 29, 70569 Stuttgart, Germany \\ ${ }^{2}$ Universitätssternwarte Oldenburg, Institute of Physics and Department of Medical Physics and Acoustics, \\ Carl von Ossietzky University, 26129 Oldenburg, Germany \\ ${ }^{3}$ European Space Agency, ESA/ESTEC, Keplerlaan 1, 2201 AZ Noordwijk ZH, the Netherlands \\ ${ }^{4}$ Chair of Astronautics, Technical Univ. Munich, Boltzmannstraße 15, 85748 Garching, Germany \\ ${ }^{5}$ International Meteor Organisation, Abenstalstr. 13b, 84072 Seysdorf, Germany \\ Correspondence to: Thomas Albin (albin@irs.uni-stuttgart.de)
}

Received: 18 August 2016 - Discussion started: 24 October 2016

Revised: 30 January 2017 - Accepted: 1 February 2017 - Published: 27 February 2017

\begin{abstract}
In this paper, we analyse the technical biases of two intensified video cameras, ICC7 and ICC9, of the double-station meteor camera system CILBO (Canary Island Long-Baseline Observatory). This is done to thoroughly understand the effects of the camera systems on the scientific data analysis. We expect a number of errors or biases that come from the system: instrumental errors, algorithmic errors and statistical errors. We analyse different observational properties, in particular the detected meteor magnitudes, apparent velocities, estimated goodness-of-fit of the astrometric measurements with respect to a great circle and the distortion of the camera.

We find that, due to a loss of sensitivity towards the edges, the cameras detect only about $55 \%$ of the meteors it could detect if it had a constant sensitivity. This detection efficiency is a function of the apparent meteor velocity.

We analyse the optical distortion of the system and the "goodness-of-fit" of individual meteor position measurements relative to a fitted great circle. The astrometric error is dominated by uncertainties in the measurement of the meteor attributed to blooming, distortion of the meteor image and the development of a wake for some meteors. The distortion of the video images can be neglected.

We compare the results of the two identical camera systems and find systematic differences. For example, the peak magnitude distribution for ICC9 is shifted by about $0.2-$ 0.4 mag towards fainter magnitudes. This can be explained by the different pointing directions of the cameras. Since
\end{abstract}

both cameras monitor the same volume in the atmosphere roughly between the two islands of Tenerife and La Palma, one camera (ICC7) points towards the west, the other one (ICC9) to the east. In particular, in the morning hours the apex source is close to the field-of-view of ICC9. Thus, these meteors appear slower, increasing the dwell time on a pixel. This is favourable for the detection of a meteor of a given magnitude.

\section{Overview and scientific objectives}

Recently, several multi-station video camera systems to observe meteors have been set up, among others, in Japan (SonotaCo et al., 2010), in Canada (Weryk et al., 2013) and in the USA (Cooke and Moser, 2012; Jenniskens et al., 2011). The Canary Island Long-Baseline Observatory (CILBO) is a double-station meteor camera set-up operated by the Meteor Research Group of the European Space Agency. It is part of the video camera system of the International Meteor Organisation (Molau et al., 2015). CILBO consists of two stations, one on Tenerife and one on La Palma. A small building with an automated roll-off roof houses a set of video cameras with image intensifiers that monitor the same volume in the atmosphere for meteors. The pointing of the cameras is such that their image centres point to a height of $100 \mathrm{~km}$ between the two islands. Analysing the same meteor as seen from both 
camera stations allows the position relative to the Earth to be derived and, with that, to the cameras.

The main scientific goals of the set-up are as follows:

a. To study physical and chemical properties of meteoroids and, taking into account the modifications of the meteoroid properties during their flight in the solar system, to constrain the physical and chemical properties of their parent body.

b. To study the variability of the background dust flux in the Earth environment during a complete year.

To fulfil these goals, the following measurements are needed: (a) flux densities of the meteors, derived from the meteor numbers per time; (b) the physical properties of the meteoroids and their distribution, derived from light curves and velocity analysis; (c) meteoroid orbits, derived from the double-station observations; (d) chemical properties of the meteoroids, derived from spectra of the meteors.

A double-station set-up is very well suited to address these points. Since the distances of the meteor to the cameras can be determined, the absolute magnitude and the velocity in $\mathrm{m} \mathrm{s}^{-1}$ can be computed. From this, the mass of the underlying meteoroid can be estimated (see e.g. Drolshagen et al., 2014; Ott et al., 2014; Kretschmer et al., 2015). This allows us to determine the flux density of meteoroids as a function of mass. From the triangulation of the positions, the 3-D trajectory of the meteoroid in geocentric coordinates is determined. Together with the velocity, the meteoroid path can be propagated backward and the heliocentric orbit of the meteoroid can be determined. From the magnitude profile of the meteor some physical properties of the object can be determined. To measure the spectra of the meteors, a second camera is installed on Tenerife, which has an objective grating.

To properly analyse all of these measurements, many biases have to be considered. Meteors of a given mass will generate more light the higher their velocity when entering the atmosphere. They will only be detected when they are above a certain brightness, which also depends on the distance to the observing camera. Because of the optical effects of the camera, they may be detectable in the centre of the field of view but not at the edges, where the camera sensitivity is lower. The higher the apparent velocity of a meteor, the more pixels are covered per unit time by the meteor, making it more difficult to detect it. The observing geometry will affect the observations - as we will show, a camera pointing to the east will record more meteors than one pointing west. This is because the east-pointing camera sees meteors from the apex direction with lower apparent velocity, increasing the dwell time and thus the meteors signal on a pixel.

In general, we distinguish between two effects - physical biases and biases in the detection system. Physical biases include effects that are independent of the detection system. For example, meteors that due to their orbital elements do no intersect with Earth's orbit need to be estimated for mod- elling purposes. This paper deals with the latter, the detection system and with geometrical aspects. This affects the detectability of meteors and biases the resulting brightness and velocity distributions depending on the camera system's set-up, settings and its pointing. The following section gives more background on the technical aspects of the system. We first describe the set-up and then summarize all the expected errors.

\section{Set-up, data flow and methods}

\subsection{CILBO overview}

A detailed overview of the set-up is given in a previous paper (Koschny et al., 2013). In this paper, we focus on the camera and the detection system, with an emphasis on their technical performance. Figure 1 shows a photograph and a block diagram of one of the cameras. It consists of the following main elements: (a) an objective lens-type Fujinon, $25 \mathrm{~mm}$ f/0.8; an image intensifier-type DEP1700 with a fibre-coupled 2/3" CCD sensor read out via a Teli CS8310BCi video camera. The resulting field of view is roughly $28^{\circ} \times 22^{\circ}(\mathrm{H} \times \mathrm{V})$.

In the following, we are analysing data from two cameras, called ICC7 (on Tenerife) and ICC9 (on La Palma). "ICC" stands for Intensified CCD camera. Both cameras are identical. They point to the same volume in the atmosphere, between the two islands. Thus their pointing azimuth is roughly opposite; the pointing elevation is similar but not quite identical.

\subsection{Data flow}

The video cameras continuously record the night sky. With a field of view of approximately $600 \mathrm{deg}^{2}$, CILBO covers an area of around $3000 \mathrm{~km}^{2}$ at an altitude of $100 \mathrm{~km}$, where most meteors appear. The camera delivers a PAL (phase alternating line) video stream via a professional frame-grabber card (Matrox Meteor II) to a personal computer. The video signal is searched in real time for meteors using the software MetRec (Molau, 1999). MetRec analyses downsampled images with a resolution of $384 \times 288$ pixel $^{2}$ and 8 bit dynamical range. Later, we will show both full-resolution data and downsampled data, depending on the context.

MetRec generates a background noise image which is subtracted before the detection. The detection algorithm itself is described in Molau (1999, 2014). The software searches for brightness peaks in the background-subtracted images. It checks whether these peaks move on a great circle from one frame to the next.

For each frame of a detection, MetRec records the total digital number of the event on the detector and the position of its photometric centre. For each detected event, it stores a sum image, an animation of the event and a file containing detailed information on the event. 

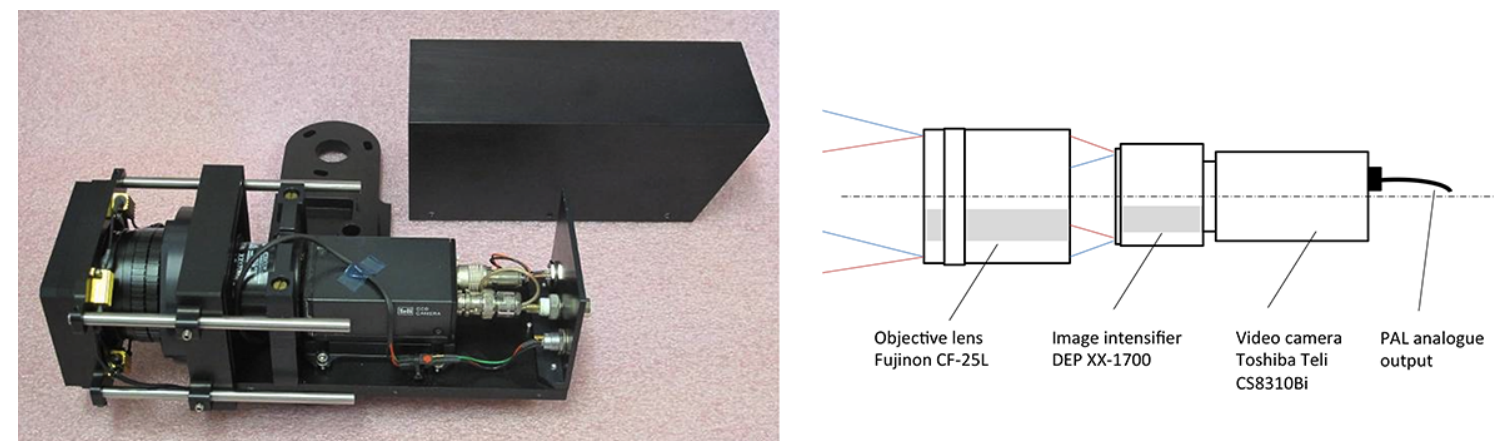

Figure 1. Photograph and sketch of the video cameras, called ICC (Intensified CCD camera).

For each night, MetRec saves all files in a daily directory. The data for ICC7 and ICC9 are stored in individual paths. The detailed information of each meteor is saved in an individual ASCII file with the extension *.inf, henceforth called "information file". Additionally, MetRec saves a log file that contains, e.g. the used detection parameters, the used reference file which contains the astrometric information of the stars and additional information of a recorded meteor.

The complete content of an information file is, for each frame where the meteor was detected, frame number, precise time taken from the computer clock, magnitude of the event, position of the photometric centre in coordinates relative to the detector and in celestial coordinates and fitted coordinates as described in the following paragraph. An example information file can be found in Koschny et al. (2013).

In addition to the information for each individual meteor, we use the log file entries in this paper to characterize the system behaviour. This file provides additional information for each detected meteor.

The automated event detection runs every clear night, controlled by a scheduling software as described in Koschny et al. (2013). At the end of the night, the data are uploaded to a central server for further processing. On the next day, the data for each night is visually inspected and false detections are deleted. The data are submitted on a monthly basis to the video archive of the International Meteor Observation, where a peer-review process ensures good data quality. All data are available and searchable via the Virtual Meteor Observatory (Barentsen and Koschny, 2008, http://vmo.imo.net).

MetRec allows us to manually compare a grabbed image with a star chart to produce a so-called "reference star" file. With this file MetRec can convert the relative positions together with the time of the event to right ascension and declination. The "referencing" process also generates a calibration file to convert pixel values to stellar magnitude. This process is typically done only when the camera pointing has changed.

MetRec attempts to correct any measurement errors in the position determination. It takes the originally measured right ascension and declination values and fits them to a great circle. The measured points are projected onto this great circle.
In a next step, MetRec shifts the points on this great circle to be equally spaced. For longer meteors ( $>7$ frames), MetRec shifts the points to match a distribution following a 2 nd-order polynomial.

If a second meteor appears during the same second as a previous on, an additional log entry with the same time stamp is saved. However, the corresponding information file with the astrometric information is overwritten and lost.

\subsection{Expected errors}

\subsubsection{Overview}

In the later sections of this paper, we will present some findings on different parameters measured by the system. Then we will draw conclusions on how important the different biases are and which ones can be corrected. In summary, we expect the following errors.

\subsubsection{Instrumental errors}

a. The mechanical/thermal instability of the mounting: due to thermal effects, the precise pointing position of the camera may change. This is a systematic error affecting the position measurement of the meteor.

b. The lens and possibly also the image intensifier generate a drop-off caused by both vignetting and the tangent effect at larger distances to the centre of the field of view. This is a systematic error affecting the detectability of a meteor.

c. Due to the projection of the celestial sphere on the flat sensor surface, the system generates distortion which needs to be corrected when computing positions of the meteors. This is corrected by the 3rd-order polynomial "plate fit" performed during the measurement; however see Sect. 2.3.3 point c.

d. The sensor is read out with 25 frames per second and the readout generates noise. In addition, random noise is generated by the image intensifier. The noise statistics 
are estimated from a sequence of dark frames (no light entering the sensor system). It is random noise affecting all measurements.

e. The pixel resolution of the sensor does not precisely match the pixel format of the used PAL format (768 pixel $\times 576$ pixel) and pixels may be interpolated.

f. The sensor is an interline-transfer sensor, i.e. every second physical line on the sensor is masked and used for readout. This and the previous point will reduce the quality of the position determination of the meteor.

g. (Absolute) timing errors (offset of the computer clock): this is a systematic error that only affects the position, not the velocity. A timing error of $1 \mathrm{~s}$ would correspond to a position error in right ascension of $1 / 4^{\prime}$.

h. Distortion of the image of a meteor close to the edge of the field of view. This effect is particularly pronounced for bright meteors and it will result in errors in the astrometric position of the meteor.

\subsubsection{Algorithmic errors}

a. Wake: during the movement of the meteor it may develop a train, which shifts the photometric centre to the opposite direction of the meteor's movement. This effect will result in an apparent change in the velocity of the meteor. Typically, trains develop towards the end of the meteor, so this effect will reduce the perceived speed of the meteor towards the end.

b. Blooming: for bright meteors, so-called blooming may occur; i.e. electrons spill over from one pixel to other adjacent pixels. The shift of the photometric centre can then go in any direction.

c. The image distortion is corrected using a 3rd-order polynomial fit. In particular, towards the edges of the field of view, a 3rd order may not be good enough to properly describe the distortion. This will introduce a systematic deviation of the measured positions with respect to the real position.

d. When determining the position of a meteor, our detection software attempts to fit the positions using a linear or quadratic equation resulting in a constant and linear equation for the velocity, respectively. Due to geometric effects this may not be sufficient to describe the position and causes a deviation between the fit and the actual measured meteor position. The effect is meteor dependent, as it is affected by the length of the meteor in number of frames. Any velocity determination error may be estimated by calculating how the velocity will really change when crossing the field of view and how good the quadratic fit is. e. Meteor beginning and end: since the meteor will start or end at a random time during the exposure of the first or last frame, taking the photometric centre as the position of the meteor for this frame does not give the correct results. This is a systematic error that only affects the velocity.

f. Quantization error of position in the information files: the position of a meteor is stored as a relative position in the frame (from 0 to 1 ) with an accuracy of three decimal places only. This corresponds roughly to 0.3 pixel. If meteor positions are recomputed later in the analysis process this information is used, resulting in a quantization of the position. This is a random error which affects both position and velocity. It is meteor dependent, because meteors with more frames will be less affected.

\subsubsection{Statistical errors}

a. Statistical random error: both the position and the brightness measurements of a meteor in an individual frame are affected. This is an error due to the probabilistic nature of the event and is independent from the used instrument or its settings. It affects both position and velocity and it can be derived from the accuracy of the meteor fit that is currently investigated. It is meteor dependent, influenced by the number of frames, meteor brightness and possibly velocity.

In the following sections, we characterize the camera systems in detail. We give results on technical aspects related to camera and software (flat field effects, distortion, etc.). We then present statistics on overall distributions of different meteor characteristics (meteor length, brightness, etc.). We combine these results and provide, as a result, the means to properly de-bias the data from the cameras for scientific analysis.

\section{Results}

\subsection{Overview}

Albin et al. (2015a, b) have made a first attempt to analyse a selected number of bias effects for meteors detected simultaneously to ICC7 and ICC9. Here we expand on this work and also treat some of the data from the cameras separately. We use data from the information and the log files.

The data flow followed the description in Sect. 1. We have used a total of 51062 and 56951 information files and 925 and $913 \log$ files for ICC7 and ICC9, respectively. The analysed time range was from 13 September 2011 to $31 \mathrm{Au}-$ gust 2015.

In the following subsections, we describe different parameters of the measurements. These will be interpreted in the discussion section. 

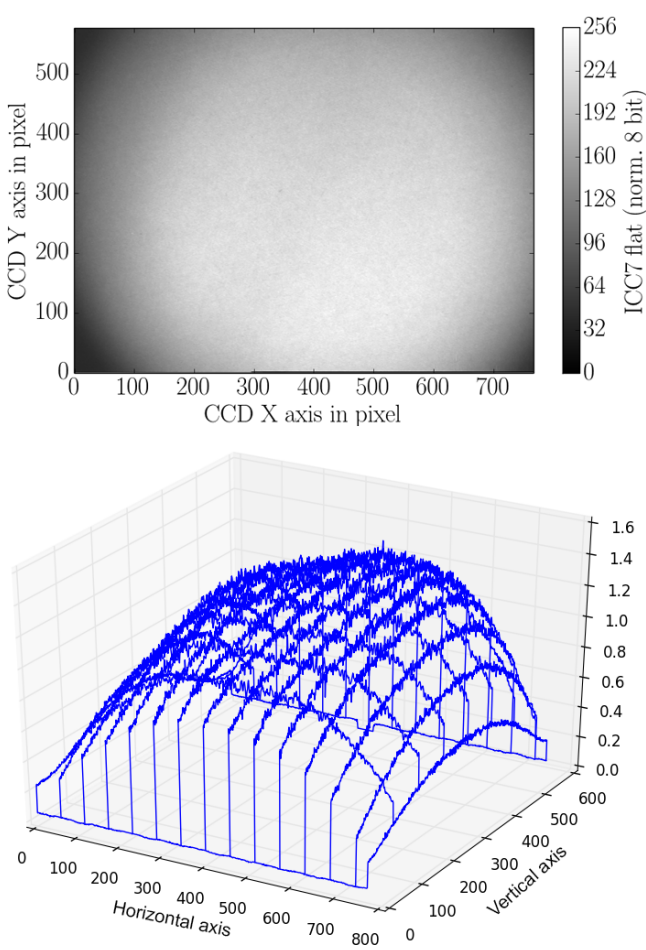

Figure 2. 8 Bit median flat of the ICC7 camera. The $x$ and $y$ axes are not downsampled, they cover the complete PAL signal. On the left, the image is shown, with the colour bar indicating the brightness of the flat field. 256 is the maximum and can be found slightly offcentre to the right due to an offset in the optical system. The bottom panel shows a wire-mesh view of the flat field. Normalized values range from 0.3 in the corners to 1.3 in the middle.

\subsection{Camera sensitivity}

We start by analysing the detection efficiency of both cameras vs. the apparent meteor velocity in pixels per second. The detection efficiency is defined as the ratio of the theoretically expected number of meteor detections on the $\mathrm{CCD}$ vs. the number of actual meteor measurements on the CCD (Albin et al., 2015a). Due to vignetting and projection effects the cameras have a sensitivity drop to the edges and corners of the CCD. Thus, the number of detections decreases to the edges due to the lower signal-to-noise ratio $(S / N)$ of the meteor, which results in less detections by MetRec. In other words, the detection efficiency would be 1 if a meteor of a given magnitude and velocity had the same $S / N$ over the complete field of view.

Figure 2 shows the flat field of the ICC7 system. The flat field of ICC 9 looks similar. The image is an 8 bit median stack of about 10 individual images, recorded when thin fog provided a rather homogeneous sky background. The grey bar indicates the corresponding normalized brightness. It can be seen that the intensity drops to the edges and corners of the CCD. An optical system with no vignetting or projection effects would lead to a uniformly shaped distribution

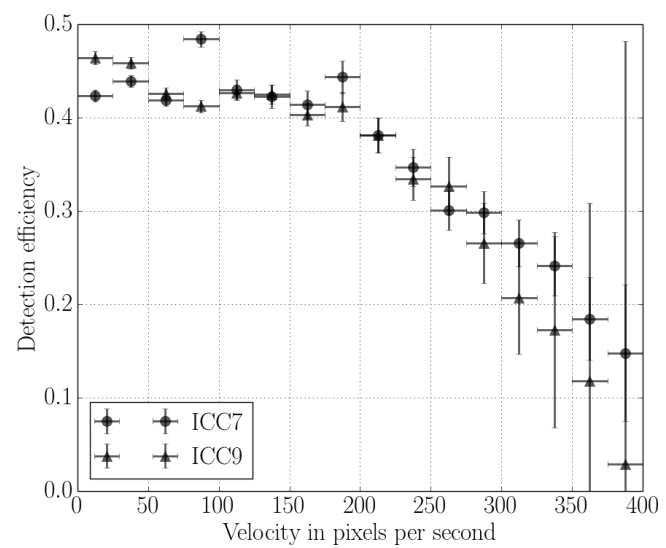

Figure 3. Detection efficiency vs. the downsampled velocity of a meteor in pixels per second. A detailed description of the detection efficiency can be found in Albin et al. (2015a).

and a detection efficiency of 1 . To compute the theoretically expected number of measurements we take the part on the CCD with the highest detection density and extrapolate this value for the complete CCD. A detailed description can be found in Albin et al. (2015a), who also computed the detection efficiency for the CILBO system depending on the meteor brightness. They found that the detection efficiency is at around 0.55 for meteors with a brightness down to $4.5 \mathrm{mag}$ and drops down to 0.45 and lower for fainter meteors. This means that the meteor cameras detect only half of the meteors which it would be able to detect for an evenly illuminated sensor.

Figure 3 shows the detection efficiency vs. the meteor velocity in pixels per second. For the analysis, we use the filtered velocity data set from the information files. The data set has been divided into bins of 25 pixel s$^{-1}$. For each bin, the theoretical and actual number of meteor detections has been computed as in Albin et al. (2015a). The plot shows the detection efficiency from 0.0 to 400 pixel s$^{-1}$. For very large velocities the number of data points decreases, increasing the shown standard deviation of the detection efficiency. It can be seen that the detection efficiency is between 0.4 and 0.5 for meteors ranging from 0.0 to 200 pixel s$^{-1}$. Then, the detection efficiency decreases approximately linearly for higher velocities.

The pixel dwell time of a meteor is inversely proportional to the apparent meteor velocity on the CCD. Consequently, a higher meteor velocity decreases the $S / N$ for a given meteor magnitude. The decreasing sensitivity to the edges and corners due to the projection effects result in a smaller effective detection area on the CCD for higher-velocity meteors. This can explain the lower detection efficiency for fast meteors.

The shown effects and the detection efficiency function as shown in Albin et al. (2015a) are necessary to de-bias the mass distribution of the meteors that is correlated to the 


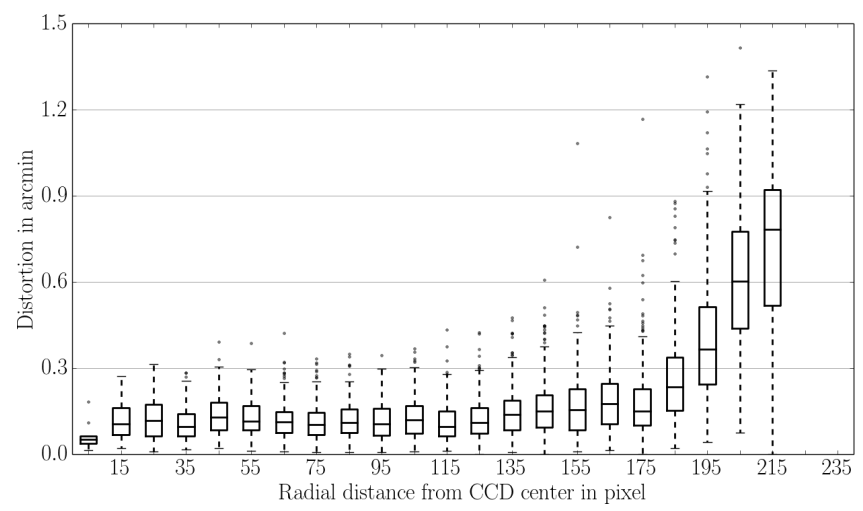

Figure 4. Box plot of the ICC7 distortion. The difference between actual position and CCD position is shown in arcminutes vs. the radial distance from the centre of the $\mathrm{CCD}$. Each box plot contains the data of the a 10-pixel-wide bin.

brightness measurements. Additionally, the determined flux needs to be corrected by at least a factor of 2 .

\subsection{Meteor velocity measurement bias}

Albin et al. (2015b) described the velocity profiles of several simultaneously detected meteors with the CILBO camera set-up. For the analysis they used the geocentric velocity in $\mathrm{km} \mathrm{s}^{-1}$ determined by the MOTS3 software package for computing trajectory data of double-station meteor cameras (Koschny and Diaz del Rio, 2002). Due to the atmospheric drag a meteoroid decelerates during the atmospheric entry. We found that $40-45 \%$ of all meteors seem to have an increased velocity between the first and second velocity measurements. This cannot be explained by Earth's gravitational attraction. The effect is an observational bias of the camera system. Both cameras are operated with a rate of 25 frames per second and a video frame length of $40 \mathrm{~ms}$ respectively. The measurable beginning and ending times of a meteor do not necessarily correspond to the video frame length of $40 \mathrm{~ms}$. Consequently, it may appear in the data set that the meteor covers a smaller distance at the beginning and end of a recording. The end part of the meteor overlaps additionally with the deceleration effect. Thus, to compute a proper initial geocentric velocity from a continuously operated double station meteor network, the distance between the first and second video frames should not be used for the velocity computation. The last velocity value should not be used for the same reason. As a result, no good velocity can be determined for meteors recorded on 3 frames only. To obtain two velocity measurements, the meteor has to be recorded on 5 frames.

\subsection{Accuracy values and optical distortion}

We generated optical distortion maps to determine the astrometric deviations of the real star positions relative to their expected positions according to the 3 rd-order polynomial plate

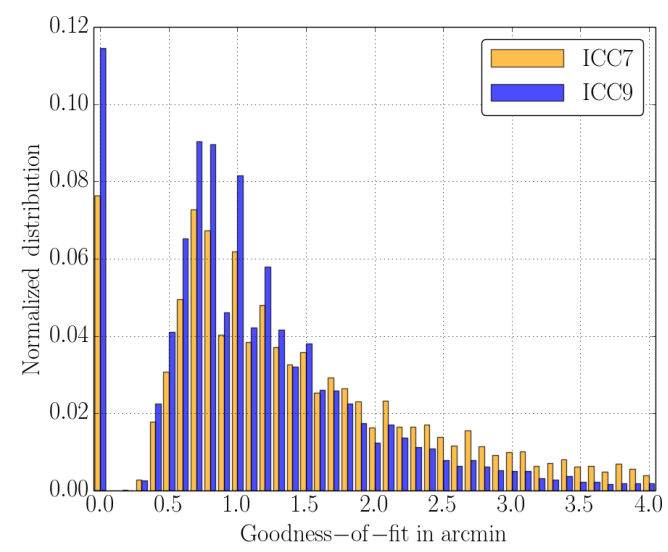

Figure 5. Normalized distribution of determined goodness-of-fit in arcminutes. The orange and blue bars show the distribution for ICC7 and ICC9, respectively. The bars are slightly off-centre and have an actual width of $0.1^{\prime}$, e.g. the first two bins show the contribution of $\left[0.0^{\prime}, 0.1^{\prime}\right)$ for ICC7 and ICC9.

fit performed by MetRec. Figure 4 shows the computed distortion distribution for the ICC7 camera. The distortion is shown by plotting the deviation of the real measured star position against its expected position determined by the plate fit. It is given in arcminutes and is plotted against the radial distance from the CCD centre in downsampled pixels. The data are summarized in bins of 10 pixels and visualized as a box $\operatorname{plot}^{1}$. It can be seen that the distortion remains approximately constant up to a radius of 140 pixels. The corresponding median is at around $0.1^{\prime}$. With the downsampled horizontal image size of 388 pixels this corresponds to $80 \%$ of the horizontal radius; $95 \%$ of the horizontal radius are correct to $0.2^{\prime}$. Due to the distortion of the optical system, the values worsen to the corners up to $0.75^{\prime}$. In conclusion, position measurements of meteors more than about $80 \%$ away from the field centre should be used carefully.

Since the ICC9 distribution looks similar, only the ICC7 data are shown. We will see that other astrometric errors are larger and conclude that at least for the inner $90 \%$ of the field of view, errors due to insufficient distortion correction can be neglected.

\subsection{Measured astrometric goodness-of-fit}

For each meteor, MetRec stores a value called "accuracy" in the log file, which describes the goodness of the fit of the

\footnotetext{
${ }^{1} \mathrm{~A}$ box plot is a way to visualize non-Gaussian distributions. It uses the so-called median and the interquartile range (IQR). The median is the point where a distribution is divided into two equalsized sets. The 25- and 75-percentile are the lower and upper limit of the IQR; the IQR contains $50 \%$ of the data around the median. In a box plot, the median is shown as a horizontal solid line in a box; the box itself corresponds to the IQR. The dashed line has a length of 1.5 IQR. Data points outside the IQR are plotted as crosses or grey circles.
} 


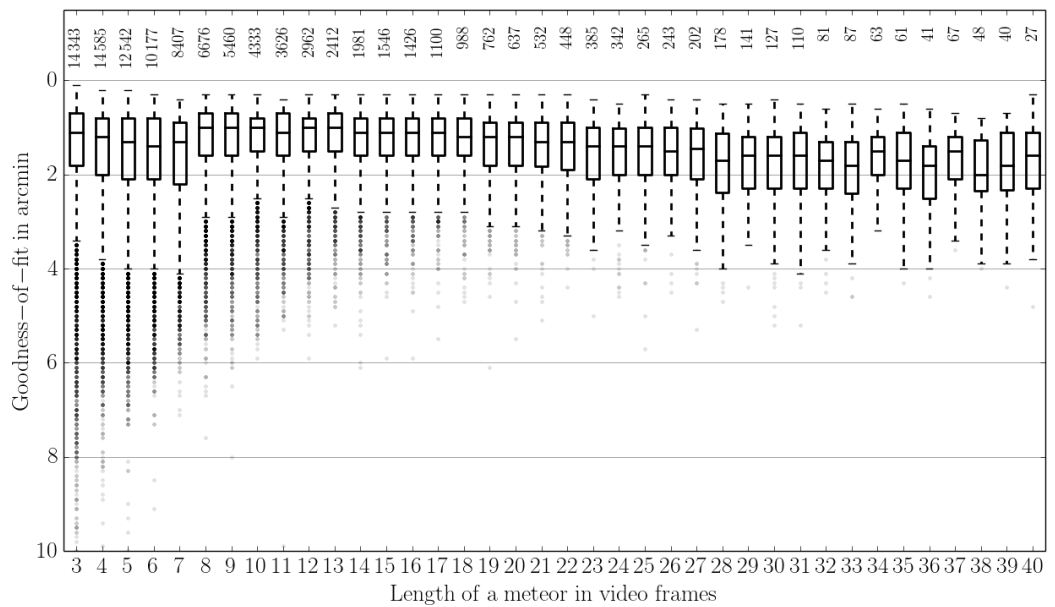

Figure 6. Goodness-of-fit vs. frame length. The box plots show the median, interquartile range (IQR) and $1.5 \cdot \mathrm{IQR}$. The numbers on the top show the number of data points for each bin.

individual meteor positions relative to a great circle in the sky. We will henceforth refer to this as "goodness-of-fit". The value is given in arcminutes and is the root mean square of the deviations of individual meteor position measurements to the projections on a least-square great circle line. The smaller the value, the better the fit. This section analyses the recorded accuracies.

Figure 5 shows the normalized goodness-of-fit distribution based on all meteor observations for ICC7 (orange or bright bars) and ICC9 (blue or dark bars). "Normalized" means that the sum of all histogram bars is 1 . The distribution plot is shown from 0.0 to $4.0^{\prime}$ with a bin width of $0.1^{\prime}$. This corresponds to the current accuracy resolution of MetRec. The maximum values are around $10^{\prime}$, but less than $3 \%$ of the data are above $4^{\prime}$ (2463 values out of 73379 ). We therefore decided to not display them.

It can be seen that both cameras detect a significant number of meteors with a goodness-of-fit of $0.0^{\prime}$. Values of 0.1 and $0.2^{\prime}$ are missing completely. The log files show that ICC7 has 3899 (approximately 8\%) and ICC9 has 6527 (approximately $11 \%$ ) of all measurements with values of 0 . For both cameras, around $55 \%$ of all measurements correspond to meteors with a length of 3 frames. Around $20 \%$ correspond to a length of 4 frames, 10 and $5 \%$ to 5 and 6 frames. The remaining $10 \%$ correspond to longer meteors. A fraction of these can be explained with the fact that MetRec rounds the determined goodness-of-fit. However, most data points in this bin seem to have been falsely generated, otherwise the gap between the $0.0^{\prime}$ bin and next bin at $0.3^{\prime}$ cannot be explained. The following accuracy-related analysis therefore neglects these data points.

The median and IQR of the ICC7 and ICC9 accuracies are $\mathrm{ICC}_{\mathrm{acc}}=1.2_{-0.5}^{+0.9^{\prime}}$ and ICC $9_{\mathrm{acc}}=1.0_{-0.3}^{+0.5^{\prime}}$.

MetRec uses half-resolution images for the detection, i.e. 384 pixel $\times 288$ pixel. The obtained average goodness-of-fit

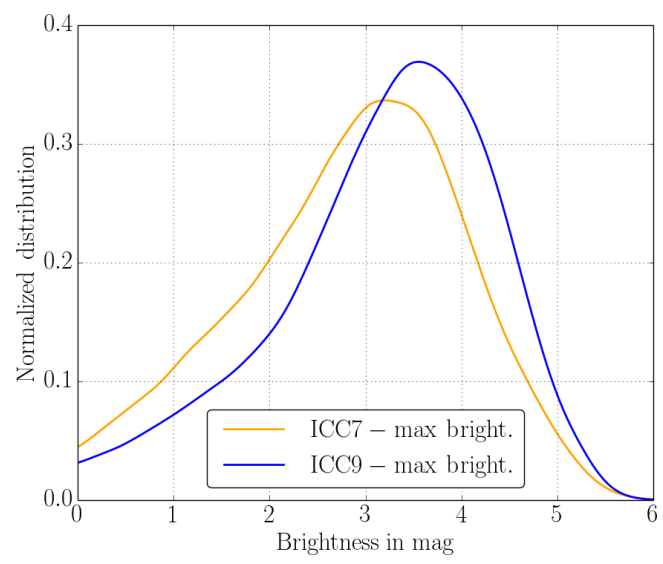

Figure 7. Normalized distribution of the peak brightness in magnitudes. The orange and blue curves correspond to the ICC7 and ICC9 camera, respectively.

is thus about $1 / 4$ pixel. Taking into account that the used sensor is an interline transfer video chip and the field of view is rather large, this result is acceptable.

When using these data to compute orbits, one can use the goodness-of-fit values to estimate, via Monte-Carlo runs, the errors of the orbital elements. A Monte-Carlo-based method to compute the astro-dynamic properties of the detected meteors is described in detail in Albin et al. (2016). To simplify this procedure, it is proposed to use an average error value as derived in the following.

Figure 6 shows a box plot of the complete accuracy data of ICC7 and ICC9 in arcminutes versus the length of a meteor measured in number of frames. All goodness-of-fit values from the log files have been used with the exception of the $0.0^{\prime}$ data. The figure shows the distribution of meteor lengths between 3 and 40 frames and the number above each box gives the number of data points in the corresponding bin. The 


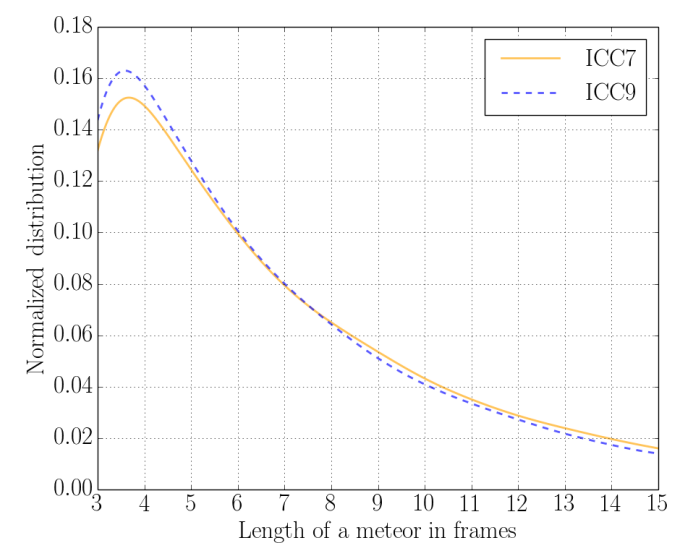

Figure 8. Normalized distribution of the recorded frames for ICC7 (solid curve) and ICC9 (dashed curve). Since MetRec's detection threshold is set to 3 frames, no meteors are recorded on fewer frames.

longest meteor recorded with CILBO is about 80 frames. For a better visualization and readability, we only show data up to 40 frames. For higher values, the total number of measurements drops further and does not allow any statistical conclusions. It can be seen that the median, the IQR and $1.5 \cdot \mathrm{IQR}$ range increase for meteor lengths of 3 to 7 frames. The median increases from 1.1 to around $1.5^{\prime}$. From 7 to 8 frames, the accuracy jumps to better values: the median drops to $1.0^{\prime}$. This is due to a setting in the MetRec fitting algorithm. Up to 7 frames, the programme uses a constant velocity value. A meteor which is recorded on 8 or more frames is fitted with a linear velocity fit which leads to a better goodness-offit, as can be seen in the changing box size between frame 7 and 8 . For meteors of length 8 to 40 frames, the accuracy worsens again slightly. The number of data points which lie outside the box plots decreases for higher frame numbers. The largest data scatter can be seen for meteor recorded on 3 frames. In some cases, the goodness-of-fit becomes as bad as $10^{\prime}$, because either the linear velocity fit was insufficient for very long meteors or outlier frames caused by noise or nearby stars were not properly detected and removed.

In conclusion, we suggest assuming a typical deviation of about $1.0-1.2^{\prime}$ to cover all uncertainties in the astrometry. This corresponds to about 1 pixel.

\subsection{Magnitude distribution}

ICC7 and ICC9 have the same technical set-up and are operated in a similar way. Items like the detection threshold and the minimum number of frames per meteor are identical. Here, we compare the measured brightness distribution of both CILBO cameras to check whether deviations in the data can be identified. For our analysis we assume that meteors appear randomly on the sky. Since some meteors either begin or end outside CILBO's field of view (FOV) or both, we consider only meteors which were completely within the FOV.

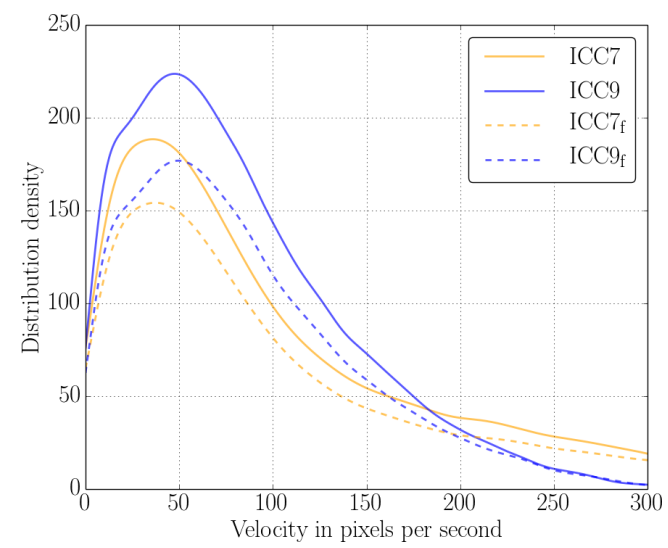

Figure 9. Distribution of the meteor velocities in pixel per second. The orange (bright) curves correspond to ICC7 and the blue (dark) curves show the ICC9 data. The solid distributions show the complete data set, containing all determined velocities. The dashed curves show the filtered velocity data set as explained in the text.

Otherwise a bias or offset in the meteors' brightness profile would affect the statistics. For the analysis we only take meteors into account that are not closer to the CCD edges than $5 \%$ of the length and width of the CCD. Thus, the data set reduces to 49494 meteors for ICC7 and 54402 meteors for ICC9 which corresponds to 97 and $96 \%$ of each individual data set, respectively.

Figure 7 shows the normalized distribution of the ICC7 and ICC9 brightness data vs. the peak brightness values in magnitudes. The orange (brighter) curve corresponds to the ICC7 data and the blue (darker) curve corresponds to the ICC9 data. The median and corresponding IQR for both cameras are $\mathrm{ICC} 7_{\text {mag,peak }}=2.92_{-0.97}^{+0.76} \mathrm{mag}$ and $\mathrm{ICC} 9_{\text {mag,peak }}=$ $3.32_{-0.88}^{+0.70} \mathrm{mag}$, respectively. This shows that ICC9 detects fainter meteors than ICC7. The brightness median difference between both cameras is 0.40 mag. We will show later that this is due to the different pointing directions of the cameras. Thus, the pointing affects the detected number of meteors for a given magnitude.

\subsection{Distribution of the length of a meteor in frames}

MetRec's detection threshold is currently set to 3 frames. With 25 frames per second this corresponds to a meteor duration of larger than $40 \mathrm{~ms}$ (starting at the very end of the exposure of the first frame, ending at the very beginning of the last one) to $120 \mathrm{~ms}$. In some rare cases a meteor with 3 frames can also have an appearance time, e.g. of $160 \mathrm{~ms}$, due to frame drops in the detection pipeline.

Figure 8 shows the normalized distribution of the length of the meteors in number of frames. The solid histogram represents the ICC7 data and the dashed histogram shows the ICC9 data. CILBO detects meteors with a length of up to $70-80$ frames. For a better data readability, here we show the distributions up to a length of 15 frames, corresponding to a 


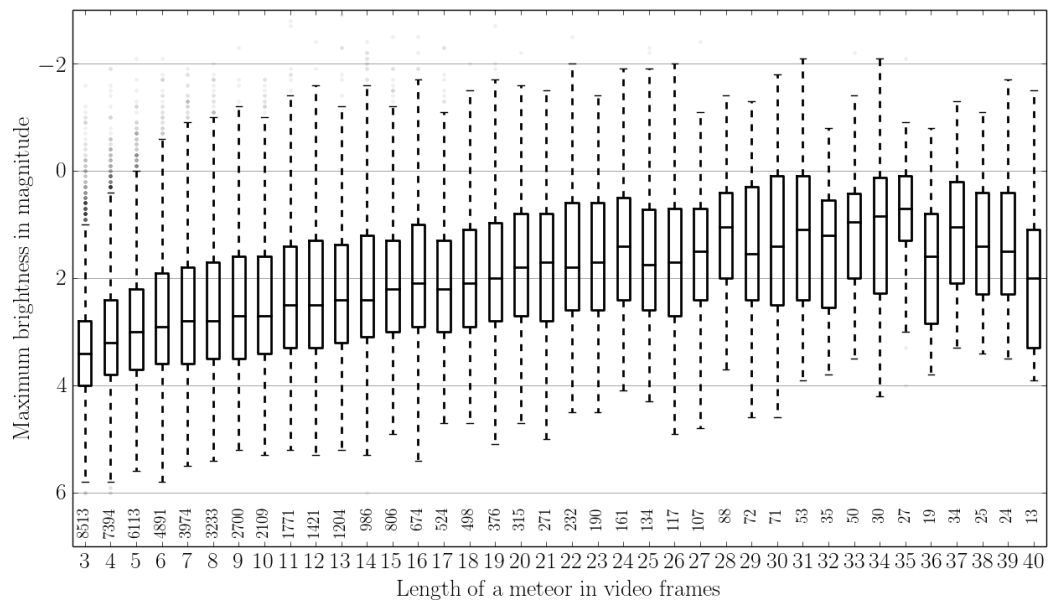

Figure 10. Maximum brightness in magnitude vs. the length of the meteor in frame numbers for ICC7. The box plot shows the median, IQR and 1.5 IQR. The number shown on the bottom indicates the number of used data points per frame bin.

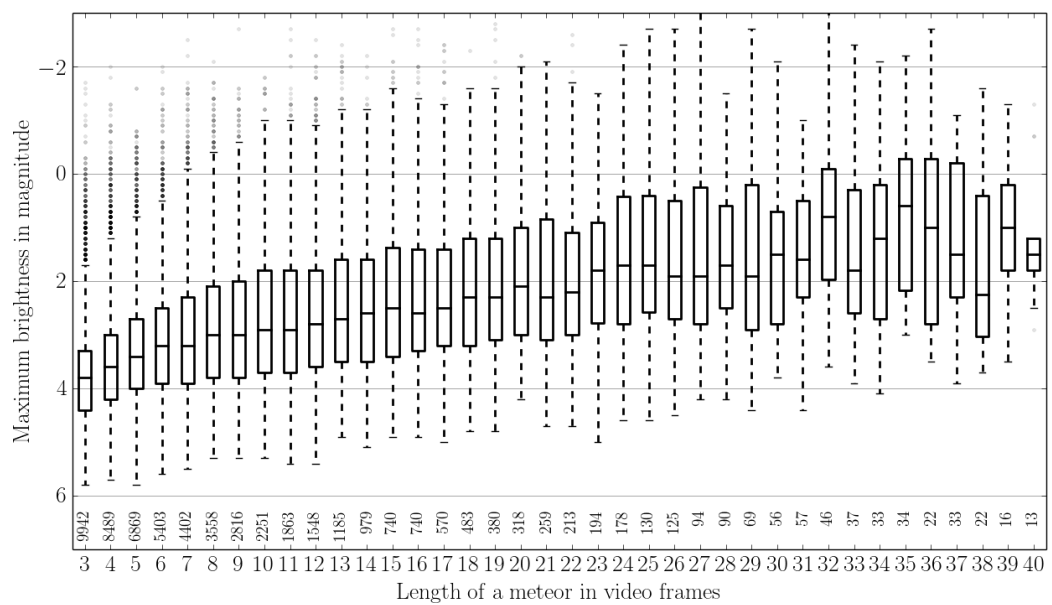

Figure 11. Maximum brightness in magnitude vs. length of the meteor in frame numbers for ICC9. The box plot shows the median, IQR and 1.5 IQR. The number shown on the bottom indicates the number of used data points per frame bin.

meteor appearance time of $0.6 \mathrm{~s}$. It can be seen that the number of meteor recordings decreases for longer events. Both distributions peak at meteors with a length of 3 frames. For increasing lengths, the number of meteors decreases faster for ICC9 than for ICC7. ICC7 detects more meteors on 3 to 7 frames than ICC9. Afterwards, the ICC7 distribution is slightly above the one of ICC9.

\subsection{Velocity distribution}

The apparent velocity of a meteor is computed from its position in each frame, assuming that the frame rate is $40 \mathrm{~ms}$. The position of a meteor is available in two coordinate systems: firstly, in a CCD-fixed system given as $x / y$ value pairs, corresponding to the horizontal and vertical position on the sensor, counted from the lower-left corner. $x$ and $y$ are normalized and range from 0 to 1 . To convert the positions in pixels, $x$ and $y$ need to be multiplied by factors of 768 and
576, respectively, which correspond to the PAL resolution. Since MetRec downsamples both axes by a factor of two we use values of 384 pixel $\times 288$ pixel for all detection-related aspects in this paper.

The second coordinate system in which MetRec provides the astrometry in is the equatorial coordinate system, where the meteor position is given in right ascension and declination. Due to optical distortions, the angular velocity distribution in degrees differs from the distribution given in CCD coordinates depending on the position in the field of view. Since this paper focuses on the technical aspects of the CILBO cameras, in the following only we consider the apparent velocity in the CCD-fixed coordinate system. For those who prefer to think in degrees per second, note that 100 pixel s$^{-1}$ will be roughly $7 \mathrm{deg} \mathrm{s}^{-1}$ with the field size of our cameras.

Figure 9 shows the density distribution of ICC 7 and ICC 9 versus the velocity in pixels per second. The solid curves 


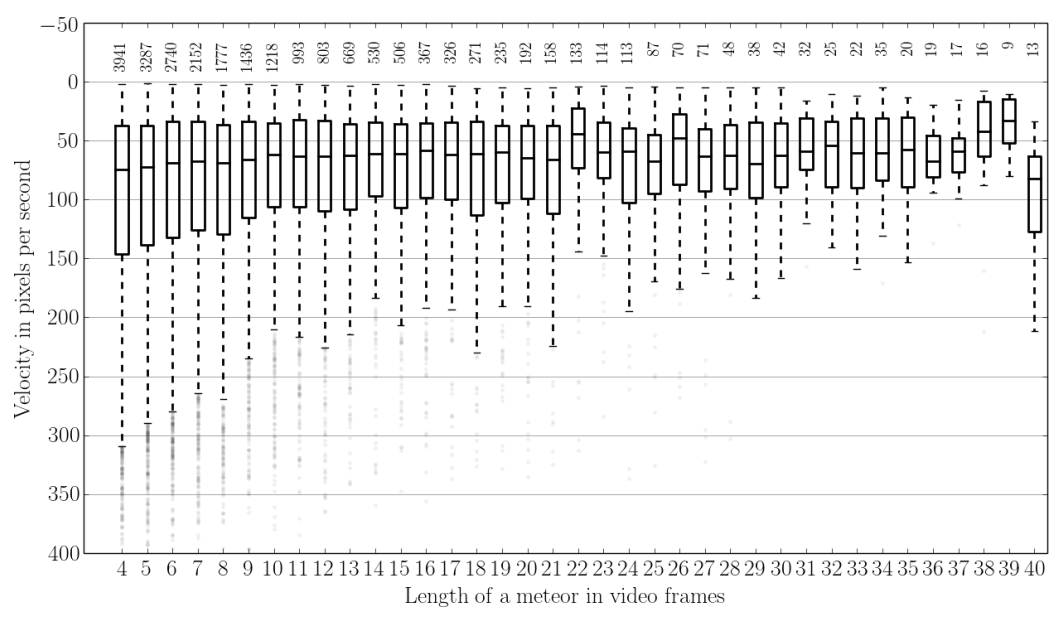

Figure 12. Apparent meteor velocity in pixels per second vs. the video frame length for ICC7. The box plot shows the median, IQR and $1.5 \cdot I Q R$. The number shown on the top indicates the number of used data points per bin.

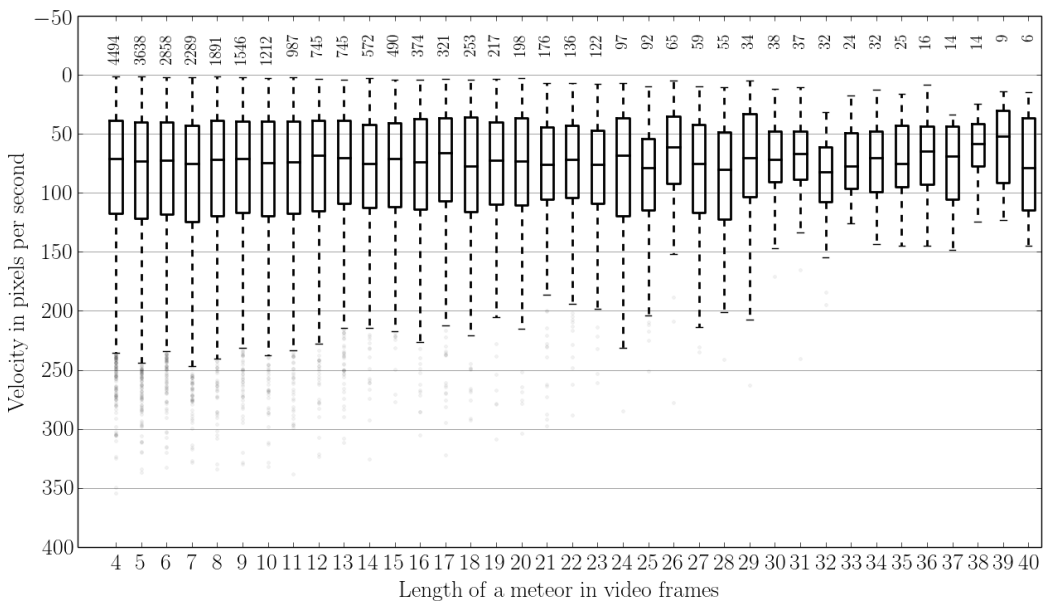

Figure 13. Apparent meteor velocity in pixels per second vs. the video frame length for ICC9. The box plot shows the median, IQR and 1.5 IQR. The number shown on the top indicates the number of used data points per bin.

are the distributions of all mean meteor velocities, where the orange (lighter) curve corresponds to ICC7 and the blue (darker) curve corresponds to ICC9 data. The velocity axis ranges from 0 to 300 pixel s$^{-1}$ (about $21 \mathrm{deg} \mathrm{s}^{-1}$ ). It can be seen that both distributions have a similar shape; however ICC9 converges faster to 0 than the ICC7 distribution. This means that ICC7 records more fast meteors than ICC9. The curve for ICC7 is flatter and crosses that for ICC9 at 195 pixel s$^{-1}$. The median and IQR (given as the error values) for ICC 7 and ICC 9 are ICC $7_{\text {vel }}=158_{-77}^{+151}$ and ICC $9_{\text {vel }}=146_{-66}^{+93}$ pixel s$^{-1}$, respectively. This shows quantitatively that the ICC7 distribution is spread wider.

Meteors appear and disappear at some arbitrary time during the exposure time of the first and last frame of a detection (see Sect. 3.4). Thus, normally the determined photometric centres of the first and last frame are shifted towards the photometric centres determined from the second and second-to-last video frame, respectively. To compute the velocity, the time interval between two frames is used, namely $40 \mathrm{~ms}$. This means that the first and last velocity determination are typically underestimated. We leave those values and call this the filtered velocity data. The dashed curves in Fig. 9 show the filtered mean velocity data sets of ICC7 and ICC9. Both dashed curves appear similar to the solid ones. The median and IQR values for both filtered data sets are $\mathrm{ICC} 7_{\text {vel, } \text {,unbiased }}=157_{-76}^{+149}$ and $\mathrm{ICC} 9_{\text {vel, unbiased }}=$ $150_{-67}^{+95}$ pixel s ${ }^{-1}$, corresponding to roughly $10 \mathrm{deg} \mathrm{s}^{-1}$.

In the following sections, we only use the filtered velocity data set if not otherwise mentioned. We suggest that velocities computed from the first and last recorded frame should not be used. 


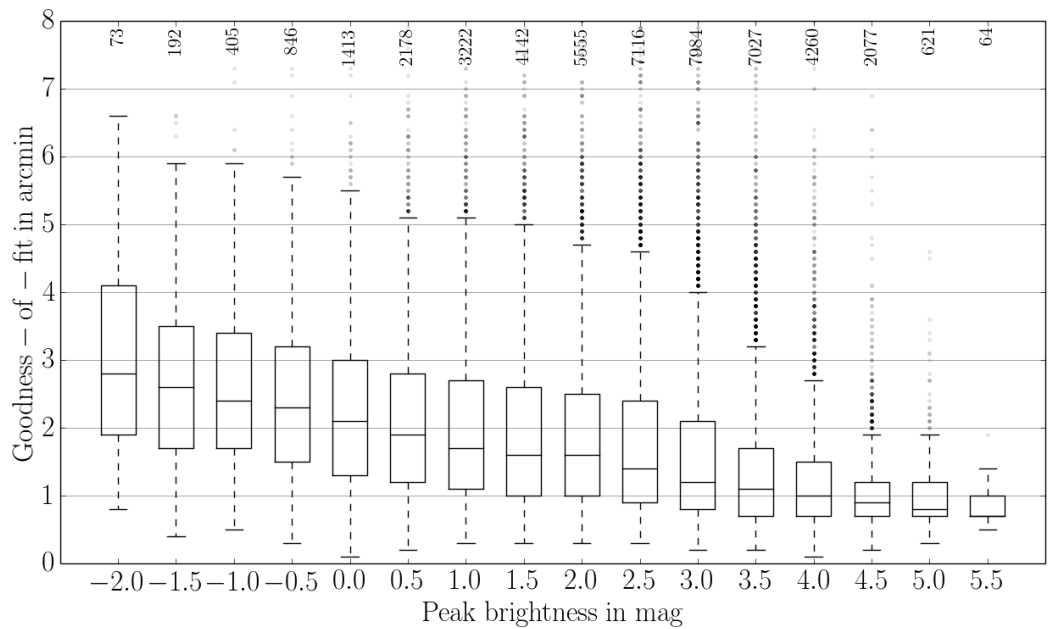

Figure 14. Goodness-of-fit vs. peak brightness in magnitude for ICC7. The box plot shows the median, IQR and $1.5 \cdot \mathrm{IQR}$. The number shown on the top indicates the number of used data points per peak brightness bin.

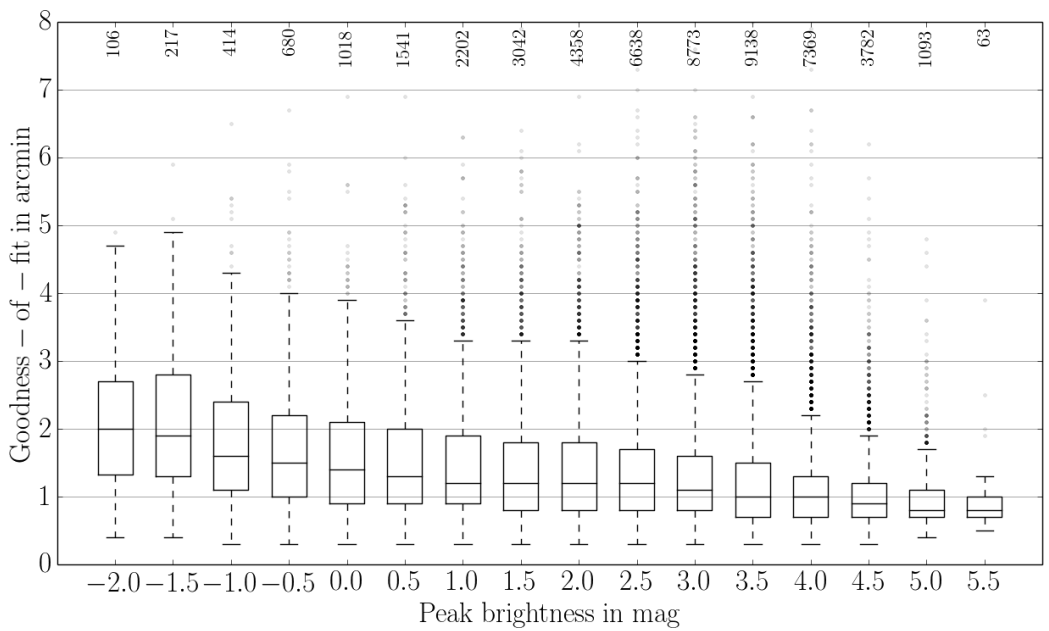

Figure 15. Goodness-of-fit vs. peak brightness in magnitude for ICC9. The box plot shows the median, IQR and $1.5 \cdot$ IQR. The number shown on the top indicates the number of used data points per peak brightness bin.

\subsection{Correlation between different measurements}

\subsubsection{Overview}

In Sect. 3.2 to 3.8 we showed distributions of different measured values like the accuracy or brightness of a meteor as determined by MetRec. Both ICC cameras are identical, but show deviations in the measured parameters. This section investigates possible correlations between certain measurements and parameters.

First, we describe the dependencies between the measurements and the recorded frame length. Afterwards we investigate possible detection time correlations. The last two subsections show some correlations with the measured brightness and determined velocities.

\subsubsection{Peak magnitude as a function of meteor length and velocity}

Figures 10 to 13 show box plots of the maximum brightness of a meteor in magnitudes and filtered mean apparent velocity in pixels per second for ICC7 and ICC9, respectively. The data are plotted vs. the length of a meteor in frames. Only meteors which were detected completely within the FOV of the cameras are considered.

The median and corresponding IQR of the brightness data for ICC7 and ICC9 show that the maximum brightness increases for longer meteors. Meteors with a length of 3 frames have a median and IQR of $3.4_{-0.6}^{+0.6} \mathrm{mag}$ for ICC7 and $3.8_{-0.5}^{+0.6}$ mag for ICC 9 . It can also be seen that the medians and IQRs of ICC9 are shifted towards fainter meteors by a factor of around 0.2-0.4 mag, consistent with Fig. 7. 


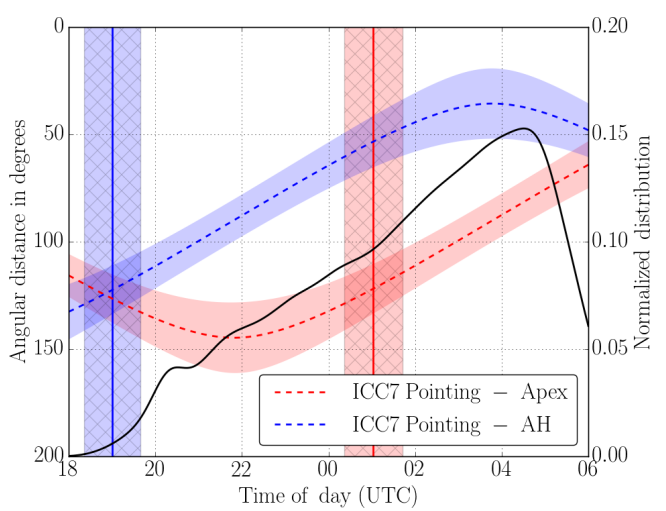

Figure 16. Angular distance and normalized distribution of detected meteors vs. the time of the day in UTC (ICC7). The red (upper) and blue (lower) dashed curves show the angular distances between the ICC7 boresight and the apex and antihelion directions, respectively. The coloured areas around the dashed lines show the yearly variations. The solid vertical lines indicate the rising time of the antihelion (blue, left) and the apex (red, right) radiants. The hatched area shows the yearly variations. The black curve corresponds to the right axis and gives the normalized number of all detected meteors.

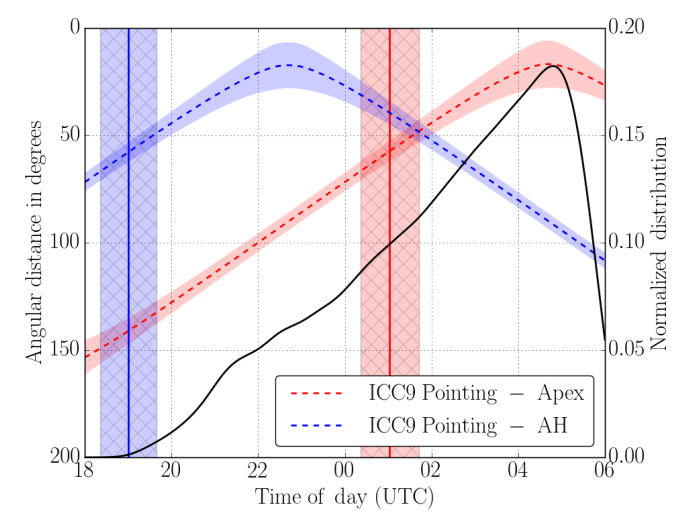

Figure 17. Angular distance and normalized distribution of detected meteors vs. the time of the day in UTC (ICC9). The red (upper) and blue (lower) dashed curves show the angular distance between the ICC 9 boresight and the apex and antihelion directions, respectively. The coloured area around the dashed lines show the yearly variations. The solid vertical lines indicate the rising time of the antihelion (blue, left) and the apex (red, right) radiants. The hatched area shows the yearly variations. The black curve corresponds to the right axis and gives the normalized number of all detected meteors.

The box plots of the velocity distributions for ICC7 and ICC9 (Figs. 12, 13) show a slight difference. Median and IQR for ICC9 are basically constant for all shown meteor lengths. The IQR ranges between 50 and 150 pixel s ${ }^{-1}$. ICC7, however, shows a decrease in the velocity for an increasing number of video frames. The maximum is at the beginning where the median is at around 75 pixel s$^{-1}$ and the IQR boundaries are at 40 and 140 pixel s$^{-1}$. The decreas-

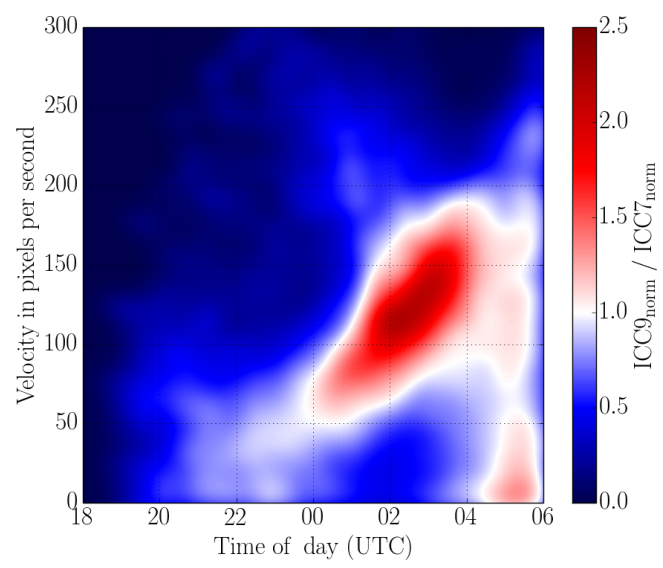

Figure 18. Ratio plot of the velocity in pixels per second of ICC9 divided by ICC7 vs. the detection time. The ratio is colour coded and given in the right colour bar.

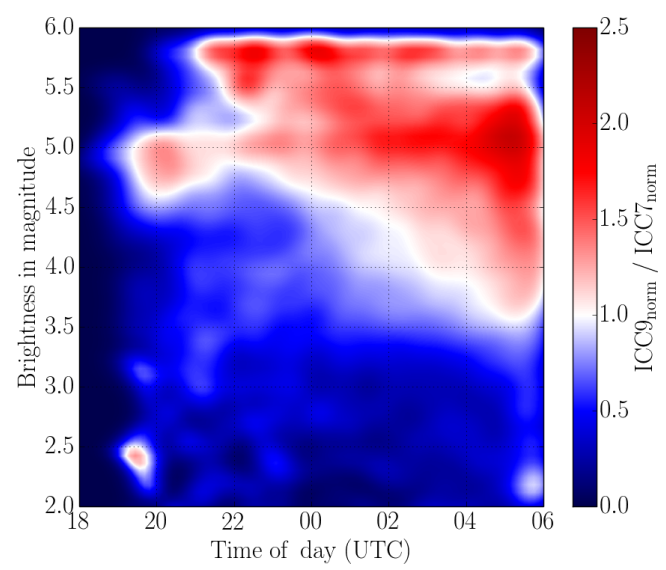

Figure 19. Ratio plot of the faintest brightness measurements of ICC 9 divided by ICC7 vs. the detection time. The ratio is colour coded and given in the right colour bar.

ing median and IQRs converge with the ICC9 data at around frame 11 .

\subsubsection{Goodness-of-fit versus peak magnitude}

Figures 14 and 15 show the measured goodness-of-fit versus the average peak brightness in mag for ICC7 and ICC9, respectively. We use all goodness-of-fit values larger than $0.0^{\prime}$. The shown figures show the data up to $6.0^{\prime}$ in a magnitude range from -2.0 to $6.0 \mathrm{mag}$. The solid line, box and the dashed lines are the median, IQR and corresponding 1.5 IQR limits. The goodness-of-fit gets smaller (i.e. better) for fainter meteors. For ICC7, the median of the goodness-of-fit at $-2.0 \mathrm{mag}$ is $3.0^{\prime}$ with an IQR of around $\pm 1.0^{\prime}$. The median decreases to $1.0^{\prime}$ at $6.0 \mathrm{mag}$. Also, the IQR range narrows towards fainter meteors. For bright meteors, the median and IQR of ICC9 is better by around $1.0^{\prime}$. Median and IQR con- 


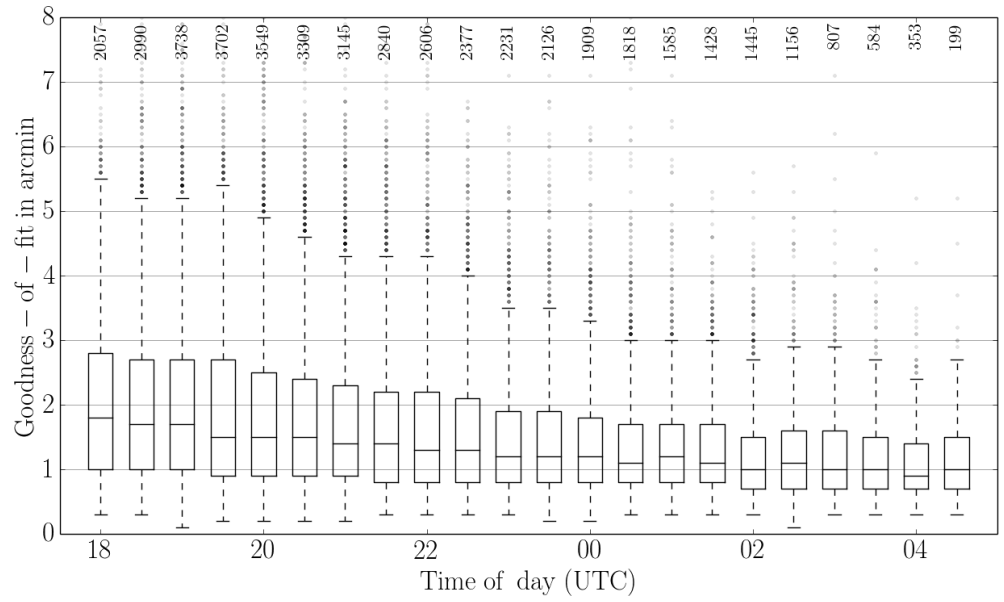

Figure 20. Goodness-of-fit vs. detection time for ICC7. The box plot shows the median, IQR and $1.5 \cdot$ IQR. The number shown on the top indicates the number of used data points per peak brightness bin.

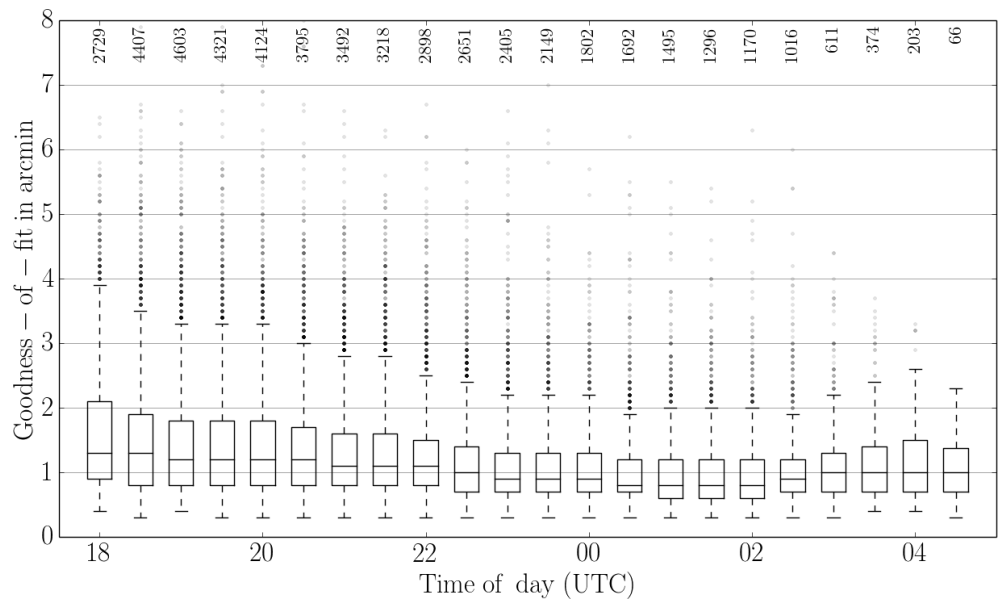

Figure 21. Goodness-of-fit vs. detection time for ICC9. The box plot shows the median, IQR and 1.5 - IQR. The number shown on the top indicates the number of used data points per peak brightness bin.

verge with the ICC7 values for fainter meteors but the IQR is slightly broader.

As mentioned in Sect. 2.3.3 point b, bright meteors overexpose the CCD pixels. This leads to blooming which results in an additional broadening of the meteor on a single video frame. Another effect may be that bright meteors are more likely to display a wake (Sect. 2.3.3 point a). Due to these effects the photometric centre cannot be determined correctly, which leads to a larger position determination error for brighter meteors.

\section{Discussion}

Even though both cameras are identical from a technical point of view, ICC9 detects fainter meteors. We argue in the following that this is a geometrical effect and can be explained by the camera pointing direction.
Both camera boresights intersect between Tenerife (ICC7) and La Palma (ICC9) at an altitude of $100 \mathrm{~km}$. Thus, ICC7 is pointing roughly to the west and ICC9 to the east. The elevations of the boresights with respect to the horizon are approximately $53^{\circ}$.

In Figs. 16 and 17 we plot the angular distance between the camera boresights and the apex and antihelion (AH) directions for the time frame 18:00 to 06:00 UTC. The red dashed line is the angular distance to the apex, the blue dashed line to the antihelion direction. The shaded areas around the lines indicate the annual variation. The black vertical lines indicate the rise times of antihelion (blue, left line) and apex (red, right line). Again, the shaded area indicates the annual variation. The thick black line is the normalized distribution of the observed meteors as a function of time during the night.

The antihelion point rises shortly after sunset, the apex direction after midnight. Since ICC7 is pointing towards the 

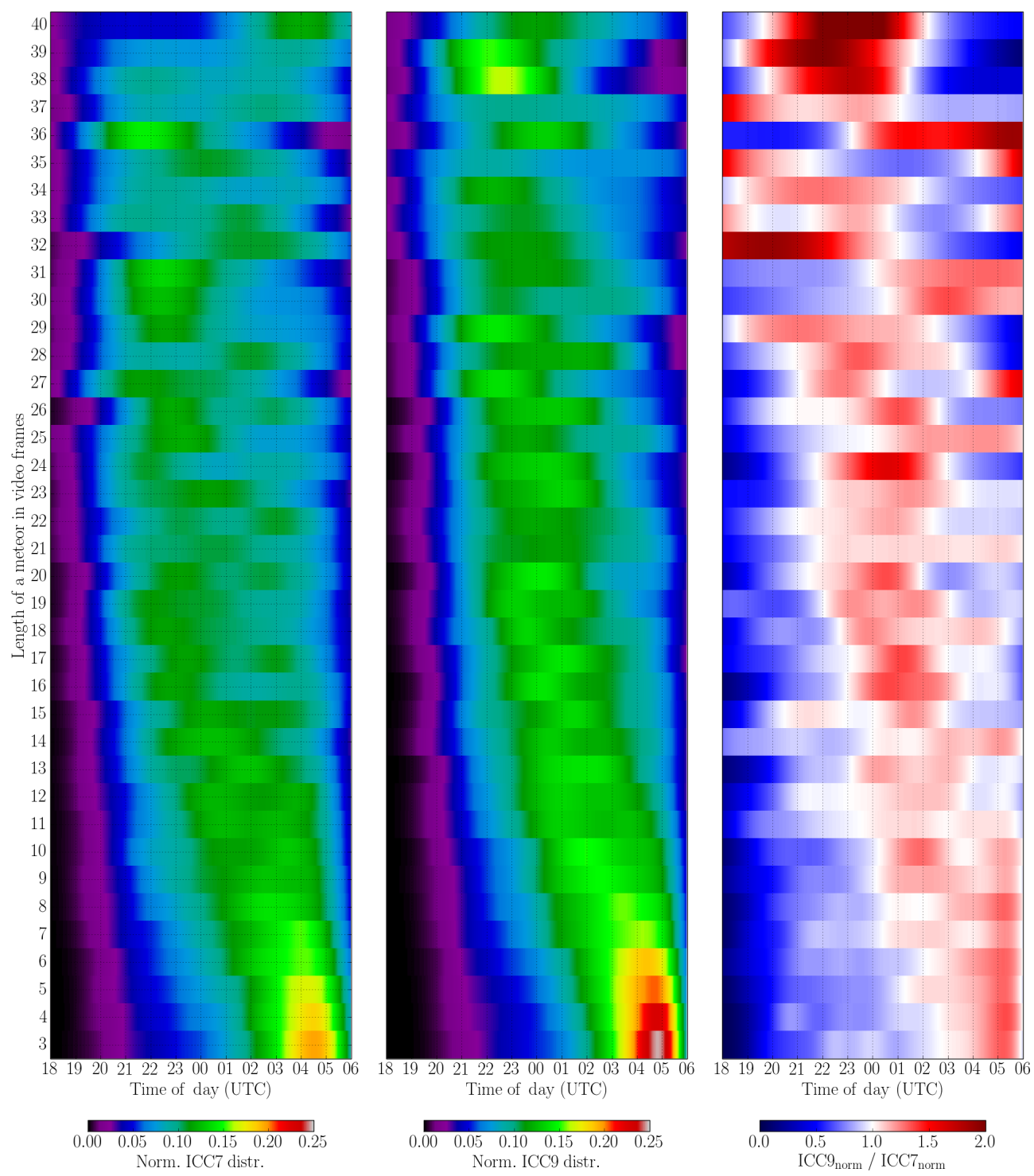

Figure 22. Ratio plot of the distribution of the normalized length of a meteor in frames of ICC9 divided by ICC7. Each frame distribution is shown vs. the detection time. The ratio is colour-coded, with the values given in the bottom colour bar.

west, its angular distance to the apex point is always much larger than for ICC9.

Figure 18 shows the ratio between the number of meteors for a given apparent velocity of ICC9 to ICC7 using a kernel density estimator (Pedregosa et al., 2011). This plot shows an interesting behaviour. Starting after midnight, ICC9 sees more meteors than ICC7 in the velocity range of 50 to 200 pixel s$^{-1}$. The peak moves to higher speeds during the night. After about 04:00 UTC, ICC9 also detects more meteors for low velocities. We explain this by the distance of the camera boresights to apex and antihelion sources. The apex is very close to the boresight of ICC 9 in the morning hours, thus the apparent velocity of these meteors is low. Since the relative speed to the Earth is high, more meteoroids of a given mass will become visible as they generate brighter meteors.
The larger number of slow meteors in ICC 9 also explains Fig. 19. Since the meteors are slower, they spend more time on a pixel and fainter meteors can be detected. This is an important finding to derive scientific conclusions, e.g. determining flux densities. The limiting magnitude determined for stars will be identical for identical systems, no matter where the camera is pointing. However, the detection threshold for meteors will be different.

In Figs. 14 and 15 we showed that the goodness-of-fit is a function of the magnitude. Since the magnitude distribution changes over the night, the goodness-of-fit also will change over night. This is illustrated in Figs. 20 and 21. The goodness-of-fit is best during the evening hours and gets worse towards the morning. The solid line indicates the median value, the dashed lines the IQRs. The values start at 
around $0.7^{\prime}$ (ICC7) and 1.0' (ICC9) and decrease over the night. We claim that this is a result of the variable radiant distance and the changing magnitude distribution over the night.

Figure 22 shows three plots of the normalized length of a meteor in frames versus time for both ICC7 and ICC9, plus the ratio between two distributions. For each frame length bin, the integral of the distribution is 1 . The colour map limits are the same for both cameras so that the differences between the camera systems can be visualized. It can be seen that both distributions show similar evolutions over time. Longer meteors are dominantly present during the evening and midnight hours and short meteors appear mostly during the morning hours. However, the distributions of ICC7 are spread wider than the distributions of ICC9. The ratio indicates a higher contribution of short meteors for ICC9 by a factor of up to 2. We explain this again by the apex meteors. ICC 9 points closer to the apex than ICC7, in particular during the morning hours. Thus apex meteors appear shorter in ICC9.

\section{Conclusion}

In Sect. 2.3 we have listed the expected errors and biases from the instrument itself, the measurement pipeline and statistical sources. Here we map the findings of the previous section to these errors.

Mechanical/thermal stability: any mechanical/thermal instability would result in a shift of the field of view relative to an Earth-fixed direction. This would shift the measured position of a meteor. When visually inspecting the data, MetRec allows us to overlay the expected star positions with the real image. This was done regularly, and such a shift was observed in very rare cases towards the morning hours. It was typically less than 2 pixels. Since it only occurred in a few nights, it was not considered in this analysis and would deserve further study.

Brightness drop-off: the drop-off of brightness towards the edges of the optical system results in a loss of about $55 \%$. This will be an important effect when computing flux densities using the limiting magnitude of the system - the detected meteor numbers really are a function of the position in the field of view. The drop-off is larger than what would be expected from pure geometrical effects. It is assumed that this is an effect of the image intensifier. For non-intensified systems, we would expect this effect to be less severe.

Astrometric accuracy: the measurement accuracy of meteor positions (astrometry) is influenced by a number of the listed errors. Figure 4 shows the deviation between measured star positions and the expected position as determined by the 3rd-order polynomial plate fit performed by the detection software. It is below $0.2^{\prime}$ up to a distance of about $90 \%$ of the diameter of the field of view. When analysing the goodnessof-fit of individual measurement points relative to the fitted great circle of the meteor's path, errors are larger. Figures 5 and 6 show that typical errors are around 1 to $1.5^{\prime}$, depending on the length of the meteor. We assume that these deviations come from the fact that MetRec determines the position of a meteor in a single frame by finding the photometric centre of the object. The resulting errors are listed under algorithmic errors in Sect. 2.3: a possible wake will shift the photometric centre to the back and blooming will shift the centre in an arbitrary direction, similar to the distortion of the meteor image. The possible rescaling from physical pixels to the PAL format (Sect. 2.3.3 point e) will also contribute to this result. As can be seen in Fig. 4, the deviation of the expected star positions to the real positions, based on the 3rd-order polynomial fit, stays around or below $0.2^{\prime}$ until about 175 pixels away from the centre of the field of view. For larger values the deviation starts to increase linearly. One of the possible reasons for this could be that the 3 rd order is not enough. We did not check whether a 4th-order fit would produce a better result; this will be future work.

We conclude that for our camera systems a typical error of 1 to $1.5^{\prime}$ should be assumed.

The position measurement inaccuracies will also affect the velocity determination. In addition, the first and last frame of the meteor should not be used for velocity determination, for the obvious reason that it is not known at what time during the $40 \mathrm{~ms}$ exposure the meteor appears or disappears.

In a future work we will determine possible effects of daily, weekly or seasonal temperature fluctuations. Scientific projects that will derive, e.g. flux densities from the CILBO camera system, will need to consider bias effects that have been shown in this work to un-bias and derive proper scientific conclusions form the observations.

We did not do a detailed analysis of random noise affecting the measurements. We assume that since the noise is random it does not produce any bias or shift in any of the measurements, but will only increase the scatter of the data.

We find that a major contribution to the detected brightness distribution comes from the pointing direction of the cameras. The pointing direction has to be taken into account when interpreting the detected number of meteors.

\section{Data availability}

Currently, data until 2015 are available on http://vmo.imo. net/. However we work currently on a new database with all data that additionally contains Monte-Carlo-based orbital elements for scientific purposes.

Competing interests. The authors declare that they have no conflict of interest.

Acknowledgements. We acknowledge the tireless efforts of Hans Smit and Cornelis van der Luijt (ESA/Space Science Office) 
for keeping the cameras operational. CILBO hardware and maintenance are funded thanks to the research faculty of ESA/Space Science Office. We also acknowledge the Instituto de Astrofisica de Canarias (J. Licandro) which hosts the CILBO system and provides local support.

Edited by: L. Vazquez

Reviewed by: P. Gural and one anonymous referee

\section{References}

Albin, T., Koschny, D., Drolshagen, G., Soja, R., Srama, R., and Poppe, B.: Influence of the pointing direction and detector sensitivity variations on the detection rate of a double station meteor camera, in: Proceedings of the International Meteor Conference, edited by: Rault, J.-L. and Roggemans, P., IMO, 226-232, 27-30 August 2015, Mistelbach, Austria, 2015a.

Albin, T., Koschny, D., Drolshagen, G., Soja, R., Poppe, B., and Srama, R.: De-biasing of the velocity determination for double station meteor observations from CILBO, in: Proceedings of the International Meteor Conference, edited by: Rault, J.-L. and Roggemans, P., IMO, 214-219, Mistelbach, Austria, 2015b.

Albin, T., Koschny, D., Soja, R., Srama, R., and Poppe, B.: A Monte-Carlo based extension of the Meteor Orbit and Trajectory Software (MOTS) for computations of orbital elements, in: Proceedings of the International Meteor Conference, edited by: Roggemans, A. and Roggemans, P., IMO, 20-25, 2-5 June 2016, Egmond, the Netherlands, 2016.

Barentsen, G. and Koschny, D.: The IMO Virtual Meteor Observatory (VMO): Architectural design, Earth Moon Planets, 102, 247-252, 2008.

Cooke, W. and Moser, D. E.: The status of the NASA All-Sky Fireball network, in: Proceedings of the International Meteor Conference, edited by: Gyssens, M. and Roggemans, P., IMO, 9-12, 15-18 September 2011, Sibiu, Romania, 2012.

Drolshagen, E., Ott, T., Koschny, D., Drolshagen, G., and Poppe, B.: Meteor velocity distribution from CILBO double station video camera dat, in: Proceedings of the International Meteor Conference, edited by: Rault, J.-L. and Roggemans, P., IMO, 16-22, 18-21 September 2014, Giron, France, 2014.

Jenniskens, P. Gural, P. S., Dynneson, L., Grigsby, B. J., Newman, K. E., Borden, M., Koop, M., and Holman, D.: CAMS: Cameras for Allsky Meteor Surveillance to establish minor meteor showers, Icarus, 216, 40-61, 2011.
Koschny, D. and Diaz del Rio, J.: Meteor Orbit and Trajectory Software (MOTS) - Determining the Position of a Meteor with Respect to the Earth Using Data Collected with the Software MetRec, WGN, 30, 87-101, 2002.

Koschny, D., Bettonvil, F., Licandro, J., Luijt, C. v. d., Mc Auliffe, J., Smit, H., Svedhem, H., de Wit, F., Witasse, O., and Zender, J.: A double-station meteor camera set-up in the Canary Islands - CILBO, Geosci. Instrum. Method. Data Syst., 2, 339348, doi:10.5194/gi-2-339-2013, 2013.

Kretschmer, J., Drolshagen, S., Koschny, D., Drolshagen, G., and Poppe, B.: De-biasing CILBO meteor observational data to mass fluxes, in: Proceedings of the International Meteor Conference, edited by: Rault, J.-L. and Roggemans, P., IMO, 209-213, 27-30 August 2015, Mistelbach, Austria, 2015.

Molau, S.: The meteor detection software MetRec, in: Proceedings of the International Meteor Conference, edited by: Arlt, R. and Knöfel, A., IMO, 9-16, 20-23 August 1998, Stará Lesná, 1999.

Molau, S.: MetRec - Meteor Recognition Software Version 5.2, available at: http://www.metrec.org/download/readme.txt (last access: 23 February 2017), 2014.

Molau, S., Kac, J., Crivello, S., Stomeo, E., Barentsen, G., Goncalves, R., Saraiva, C., Maciewski, M., and Maslov, M.: Results of the IMO Video Meteor Network - April 2015, WGN, Journal of the International Meteor Organization, 43, 115-120, 2015.

Ott, T., Drolshagen, E., Koschny, D., Drolshagen, G., and Poppe, B.: Meteoroid flux determination using image intensified video camera data from the CILBO double station, in: Proceedings of the International Meteor Conference, edited by: Rault, J.-L. and Roggemans, P., IMO, 23-29, 18-21 September 2014, Giron, France, 2014.

Pedregosa, F., Varoquaux, G., Gramfort, A., Michel, V., Thirion, B., Grisel, O., Blondel, M., Prettenhofer, P., Weiss, R., Dubourg, V., Vanderplas, J., Passos, A., Cournapeau, D., Brucher, M., Perrot, M., and Duchesnay, E.: Scikit-learn: Machine Learning in Python, J. Mach. Learn. Res., 12, 2825-2830, 2011.

SonotaCo, T., Molau, S., and Koschny, D.: Amateur contributions to meteor astronomy, European Planetary Science Congress 2010, 20-24 September, Rome, Italy, p. 798, 2010.

Weryk, R. J., Campbell-Brown, M. D., Wiegert, P. A., Brown, P. G., Krzeminski, Z., and Musci, R.: The Canadian Automated Meteor Observatory (CAMO): System overview, Icarus, 225, 614-622, 2013. 